\title{
El redimensionamiento del derecho internacional del trabajo
}

\section{The resizing of International Labor Law}

\author{
MIGUEL CANESSA*
}

\begin{abstract}
Resumen: Las transformaciones que viene provocando la globalización en el mundo del trabajo hacen insuficientes los esfuerzos desde los ordenamientos nacionales para abordar un fenómeno que trasciende sus fronteras. De igual modo, los esfuerzos desde la Organización Internacional del Trabajo son meritorios pero todavía incompletos porque sus Miembros son renuentes a adquirir nuevos compromisos internacionales. Frente a este panorama, son los derechos humanos laborales los que están en mejores condiciones para enfrentar los retos de la globalización.
\end{abstract}

Palabras claves Derecho Internacional del Trabajo - Derechos Humanos Laborales - globalización - comercio internacional - ius cogens

Summary: The transformations brought about by the globalization of the labour world render insufficient the national efforts to face a cross-boundaries phenomenon. Similarly, the International Labour Organization efforts are praiseworthy but incomplete because its Members are reluctant to new international commitments. In this situation, labour human rights are in the best position to face the challenges of globalization.

Keywords: International Labour Law - Labour Human Rights - globalization - international trade - ius cogens

CONTENIDO: INTRODUCCIÓN.- I. LA REVALORACIÓN DEL DERECHO INTERNACIONAL DEL TRABAJO.- II. LOS DERECHOS HUMANOS LABORALES EN EL DERECHO INTERNACIONAL GENERAL.- III. LA PROTECCIÓN INTERNACIONAL DE LOS DERECHOS HUMANOS LABORALES.- IV. REFLEXIÓN FINAL.

\section{INTRODUCCIÓN}

Desde fines del siglo pasado se viene transitando en el mundo del trabajo por un período de grandes transformaciones que han modificado sustancialmente los pilares sobre los que se constituyó el Derecho Laboral. El impacto de las nuevas formas de organización del trabajo ha supuesto un reto para la normativa laboral, acostumbrada a regular el trabajo subordinado y por cuenta ajena, ahora enfrenta el desafío de ampliar su campo de regulación hacia el trabajo autónomo y la subcontratación.

* Abogado y sociólogo de la Pontificia Universidad Católica del Perú. Doctor en Derechos Humanos de la Universidad Carlos III de Madrid. Profesor de las Maestrías de Derecho Laboral y de Derechos Humanos de la Pontificia Universidad Católica del Perú. Consultor Internacional. 
A su vez, la revolución tecnológica ha facilitado la producción a nivel global, donde la empresa-red se convierte en el paradigma de la unidad productiva. Inclusive, la independización de las fases de producción ya no solo se materializa al interior de la empresa, sino también puede materializarse externamente a ella. Así, el nuevo escenario productivo nos muestra dos tendencias que conviven simultáneamente. Por un lado, empresas que optan por subdividirse en conglomerado de organizaciones empresariales, siempre dirigidas desde una central. Por otro lado, empresas que trasladan a otras empresas fases de su producción contratándolas como subcontratistas o proveedores. Ambas modalidades dan forma al proceso de descentralización productiva, donde apoyándose en las nuevas tecnologías y el abaratamiento de los costes de transporte, las empresas rediseñan sus organizaciones en busca de obtener mejores niveles de eficiencia productiva en términos internacionales.

Simultáneamente, hay un debilitamiento del Estado-Nación provocado por la globalización de la economía. Los espacios nacionales se ven desbordados por unidades económicas que trascienden las fronteras, con lo que se pierde control sobre ellas y, en muchos casos, estas resultan siendo más fuertes que un significativo número de Estados. La empresa multinacional (EMN) se convierte en el actor preponderante de la globalización, a tal grado que logra marcar el derrotero de la acción estatal y de los organismos internacionales.

Enfrentamos un escenario en que los paradigmas del mundo del trabajo se han transformado sustancialmente, y a pesar de los esfuerzos normativos de las legislaciones nacionales, estos no pueden desde sus ámbitos de aplicación cubrir los cambios producidos en el mundo laboral. La nueva dinámica económica es especialmente trasnacional y los esfuerzos de los Estados se ven limitados por sus fronteras.

Este nuevo escenario plantea como exigencia una regulación que gobierne la dimensión laboral de la globalización económica, regulación que no puede provenir de los ordenamientos nacionales sino que más bien coloca al Derecho Internacional del Trabajo como el espacio jurídico natural donde implementarla. Esto nos conduce a preguntarnos: ¿el Derecho Internacional del Trabajo está en condiciones de enfrentar este reto? ¿Existen el marco regulador y los procedimientos de control que aseguren su cumplimiento?

El presente artículo tiene la pretensión de esbozar respuestas a estas dos interrogantes. Para ello ha sido dividido en tres secciones. En primer lugar, aborda la revaloración del ámbito normativo del Derecho Internacional del Trabajo con su expansión en otras ramas jurídicas. En segundo lugar, resalta la importancia de los Derechos Humanos Laborales en el nuevo contexto de la globalización económica. En tercer lugar, plantea un somero análisis sobre la protección internacional de los derechos humanos laborales. 


\section{LA REVALORACIÓN DEL DERECHO INTERNACIONAL DEL TRABAJO}

La visión clásica del Derecho Internacional del Trabajo (DIT) suele tener como referente principal a las normas internacionales del trabajo - convenios y recomendaciones - de la Organización Internacional del Trabajo (OIT). Sin embargo, es una visión parcial porque deja de lado a otros espacios del DIT que son tan importantes como las propias normas del organismo internacional.

Para mostrar las verdaderas fronteras jurídicas del DIT se presenta el gráfico 1 que retrata su ámbito normativo en relación con otras dos ramas jurídicas del Derecho Internacional general: el Derecho Internacional de los Derechos Humanos (DIDH) y el Comercio Internacional (CI), donde también se recogen derechos laborales. Así el gráfico representa tres círculos: el primero, en la parte superior, muestra el DIT, el segundo círculo, de la parte inferior izquierda, muestra el DIDH y el tercero, parte inferior derecha, muestra el CI.

\section{Gráfico 1: Los derechos laborales en el Derecho Internacional general}

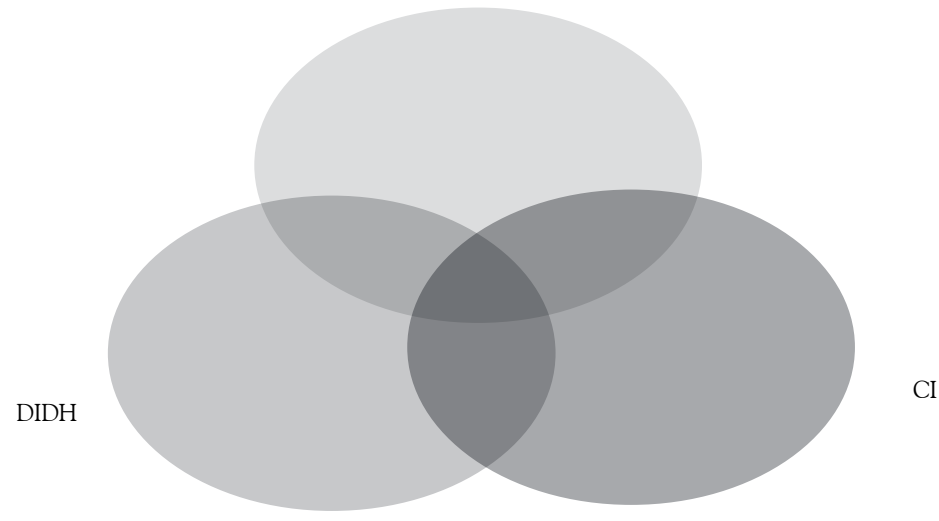

Como se ha señalado, desde la visión clásica se plantea como la regulación principal del DIT a las normas internacionales de la OIT, dejando de lado otros instrumentos internacionales que también recogen derechos laborales. La explicación de esta visión proviene de la centralidad que siempre ha ocupado la OIT desde su conformación en 1919. Partamos por reconocer que una de las principales funciones del organismo internacional ha sido la elaboración en su seno de dos clases de normas internacionales - los convenios y las recomendaciones - con el objetivo de regular desde una perspectiva internacional las relaciones laborales de sus Estados Miembros. Esa centralidad de la normativa de la OIT ha ensombrecido a las otras regulaciones internacionales en materia laboral.

EL REDIMENSIONAMIENTO DEL DERECHO

INTERNACIONAL DEL TRABAJO

THE RESIZING OF INTERNATIONAL LABOR LAW 
Las normas internacionales de la OIT han venido ampliando sucesivamente en el tiempo su ámbito normativo, contando con el respaldo de las ratificaciones de los Estados Miembros y, en un momento, el de las sentencias de la fenecida Corte Permanente de Justicia Internacional. Solo en los últimos años se ha producido un reflujo dentro del organismo internacional, pero en general las normas internacionales del trabajo cubren las más variadas materias del mundo laboral.

El segundo círculo representa el DIDH, al que, a mi juicio, los laboralistas no hemos sabido otorgar la verdadera importancia que le corresponde en la regulación del mundo laboral. Cuando revisamos los paradigmáticos estudios sobre el DIT de Wilfred Jenks o Nicolás Valticos, parecería que los instrumentos internacionales de derechos humanos que recogen derechos laborales no fueran relevantes, dada su pobre mención ${ }^{1}$.

Lo controversial de esta situación es que el DIT ha tenido una fuerte influencia sobre el DIDH desde sus inicios. No fue una sorpresa que la Declaración Universal de los Derechos del Hombre (1948) y, posteriormente, ambos Pactos Internacionales de Derechos Humanos (1966) incluyesen derechos laborales. Se entendió que el mundo del trabajo en el que se desenvuelven los seres humanos tenía que ser protegido por un conjunto de derechos laborales básicos que asegurase el respeto a la dignidad humana. Esta visión se ha mantenido constante con los posteriores instrumentos internacionales de derechos humanos - tanto universales como regionales - donde los derechos laborales siguen ocupando dicha centralidad.

La intersección entre ambos círculos - DIT y DIDH - representa a los «derechos humanos laborales» que pueden ser definidos formalmente como aquellos derechos en materia laboral consagrados en instrumentos internacionales de derechos humanos que reconocen universalmente como titular a la persona humana. Esta definición formal nos permite señalar, en primer lugar, que si un derecho laboral se encuentra consagrado en un instrumento internacional de derechos humanos se ubica dentro de esta categoría; y, en segundo lugar, que su titularidad corresponde a todos los seres humanos con independencia de su nacionalidad.

El tercer círculo representa al CI en sus dos marcos normativos: universal y regional. El primer caso se refiere a la regulación desde la Organización Mundial del Comercio (OMC). El segundo caso se refiere

1 Se plantea en el estudio de Valticos como una categoría doctrinal a los derechos humanos fundamentales, un específico grupo de derechos laborales - la libertad sindical, la prohibición del trabajo forzoso u obligatorio, y la prohibición de la discriminación- que son presentados como los más importantes dentro del listado de materias laborales adoptadas por la OIT. Sin embargo, no se justifica su importancia por su reconocimiento en instrumentos internacionales de derechos humanos dado que sería una afirmación incorrecta en tanto el listado de derechos laborales es más extenso como posteriormente veremos. 
a sus dos dimensiones: los tratados de integración (por ejemplo: la Unión Europea, MERCosur, Comunidad Andina, etcétera) y los Tratados de Libre Comercio (TLC).

Lo significativo es que existe una intersección entre estas tres ramas del Derecho Internacional, a pesar de que desde el ámbito universal del $\mathrm{CI}$-me refiero al marco regulador de la $\mathrm{OMC}$ - se ha puesto reparos a que exista una conexión entre los derechos laborales y el comercio internacional. La intersección común se ubica en el plano de los derechos humanos laborales, aunque también resulta importante resaltar que no todos estos forman parte del CI.

En la actualidad nos encontramos en un proceso de definición sobre el listado de los derechos humanos laborales que tienen un carácter vinculante en el CI. Así, en el plano de los acuerdos multilaterales o bilaterales entre los Estados, existe un proceso más consolidado en el reconocimiento de derechos laborales. Basta repasar los procesos de integración europeo y americano, así como los diferentes TLC en la región para evidenciarlo.

Sin embargo, esto no es similar en el plano universal de la $\mathrm{OMC}$, donde el rechazo al vínculo se ha mantenido y salvo los casos expresos de la protección a las personas por razones de salud y la prohibición del trabajo penitenciario - artículo XX literales b) y e) del Acuerdo General sobre Aranceles Aduaneros y Comercio (GATT) respectivamente ${ }^{2}$-, no se considera que la violación grave de los derechos laborales en un Estado justifiquen la restricción o prohibición del intercambio comercial. No obstante esto, el sistema de solución de diferencias de la OMC se ha visto abocado a analizar temas laborales, muestra de ello son los casos Amianto (Canadá vs. Francia) y Sistema General de Preferencias (India vs. Unión Europea). Esto conduce a pensar que paulatinamente la OMC se va a ver obligada a ventilar indirectamente casos laborales en su sistema de solución de diferencias, aunque rechace formalmente el vínculo entre el respeto de los derechos laborales y el CI.

Por ello, el DIT no se reduce solo a la normativa desarrollada desde la OIT, sino también implica a aquella implementada en el Derecho Internacional de los Derechos Humanos y en el CI cuando recoge derechos laborales. Esas intersecciones con las otras ramas jurídicas

2 El artículo XX, literal b) del GATT permite a los Estados establecer restricciones para proteger la salud y la vida de las personas y de los animales o para preservar los vegetales. Por ello, bajo el amparo de este literal se puede restringir el intercambio comercial internacional de las mercancías que pongan en peligro la salud de los trabajadores nacionales. De igual modo, el literal e) del artículo XX del GATT permite a los Estados establecer restricciones sobre las mercancías fabricadas en prisiones. Ambas restricciones se corresponden parcialmente con prohibiciones del Derecho del Trabajo. En el primer caso existe un vínculo entre la restricción comercial para proteger la salud de las personas y el derecho a la seguridad y salud en el trabajo. En el segundo caso la restricción comercial por el trabajo penitenciario se ubica en una de las modalidades de la prohibición del trabajo forzoso $\mathrm{u}$ obligatorio.

EL REDIMENSIONAMIENTO DEL DERECHO

INTERNACIONAL DEL TRABAJO

THE RESIZING OF INTERNATIONAL LABOR LAW 
fortalecen el contenido de estos derechos, así como los protegen internacionalmente con sus procedimientos de control. Esto último significa también que los derechos laborales son protegidos por el sistema de derechos humanos — universal y regional — y los sistemas de solución de diferencias del CI.

Se debe destacar también que dentro de esta triple intersección se ubica el ius cogens laboral, aunque no cubra por completo ese espacio. Como sabemos, las normas imperativas o de ius cogens del Derecho Internacional general son aquellas regulaciones aceptadas y reconocidas por la comunidad internacional en su conjunto, que siendo inderogables establecen la nulidad de las normas internacionales o las acciones unilaterales de los Estados que están en contradicción con ellas ${ }^{3}$.

Bajo la actual etapa del Derecho Internacional general no todos los derechos humanos laborales están recogidos por normas imperativas, en razón que tanto la jurisprudencia internacional como la práctica de los Estados han precisado que solo un segmento de ellos puede ser ubicado en este ámbito. Siguiendo este criterio, solo la prohibición de la esclavitud y de la servidumbre, así como la prohibición de la discriminación en el ámbito laboral constituyen los dos derechos humanos laborales del ius cogens laboral ${ }^{4}$. No por esto debe interpretarse que otros derechos laborales estén impedidos posteriormente a integrarse bajo esta figura normativa por medio de la práctica internacional ${ }^{5}$.

Hay que estar pendiente y de no confundir las normas imperativas del Derecho Internacional general con las normas imperativas de los ordenamientos nacionales, aunque ambas tengan como raíz común el Derecho Romano y compartan la indisponibilidad de su regulación.

En efecto, el ius cogens del Derecho Internacional se constituye sobre la aceptaciónyelreconocimientodelaprácticadelacomunidadinternacional, mientras las normas imperativas nacionales vienen establecidas por su propio ordenamiento. Esto explica el porqué determinados derechos puedan ubicarse dentro de las normas imperativas de los ordenamientos nacionales y, sin embargo, la práctica de la comunidad internacional no los haya considerado dentro del ius cogens del Derecho Internacional general. Con mayor razón, no podemos implementar una lectura que traslade mecánicamente el listado de derechos laborales establecidos en normas imperativas de los ordenamientos nacionales hacia el ius cogens del Derecho Internacional general porque lo desnaturalizaría.

3 Las normas imperativas del Derecho Internacional general se encuentran recogidas en los artículos 53 y 64 de la Convenciones de Viena (1969 y 1986).

4 Para un análisis más detallado ver CANESSA MONTEJO, Miguel F. La protección internacional de los derechos humanos laborales. Valencia: Tirant lo Blanch, 2008, pp. 517 y ss.

5 Actualmente se vienen produciendo pronunciamientos de órganos de control internacional que ubican a la prohibición del trabajo forzoso u obligatorio dentro del ius cogens. 
La importancia jurídica del ius cogens laboral consiste en que su respeto es obligatorio para los Estados tanto en el plano internacional como en su ordenamiento interno. Esto significa que cualquier norma convencional o consuetudinaria internacional y cualquier acto unilateral del Estado (una ley, una sentencia, una norma administrativa, etcétera) que esté en oposición al ius cogens laboral es nulo y carente de valor jurídico, configurándose la violación de una obligación internacional y la responsabilidad del Estado por un hecho internacionalmente ilícito.

En conclusión, los derechos laborales en el plano internacional no se reducen a las normas internacionales del trabajo de la OIT, sino que también son recogidos en instrumentos internacionales de derechos humanos y en los tratados del CI. Esto redimensiona el DIT que queda retratado a partir de las intersecciones con las otras dos ramas jurídicas.

\section{LOS DERECHOS HUMANOS LABORALES EN EL DERECHO INTERNACIONAL GENERAL}

Resulta apropiado retomar el concepto formal de los derechos humanos laborales y agregarle argumentos que justifiquen su reconocimiento normativo para superar los cuestionamientos formulados desde posiciones que rechazan los derechos laborales como componente de los derechos humanos. Estas justificaciones debidamente argumentadas permiten transitar de la formalidad conceptual hacia un contenido material de los derechos humanos laborales.

Así, los derechos humanos laborales son todos aquellos derechos en materia laboral consagrados en instrumentos internacionales de derechos humanos que reconocen universalmente como titular a la persona, respetando la dignidad humana y satisfaciendo las necesidades básicas en el mundo del trabajo. Ambas justificaciones se apoyan en las fundamentaciones axiológica y antropológica respectivamente, permitiendo que el trabajador actúe de forma autónoma y sin sufrir un daño o perjuicio grave por trabajar.

La consagración de los derechos laborales en los instrumentos internacionales de derechos humanos y en las constituciones nacionales ha sido un proceso permanente durante el pasado siglo XX. En el plano internacional se produce en dos ámbitos: el universal y el regional. El primero se remonta a la constitución de la OIT en 1919 y la normativa internacional del trabajo producida en su seno, consolidándose con la Declaración Universal de Derechos Humanos (1948) y los Pactos Internacionales de 1966; y, el segundo, en los tratados regionales de derechos humanos que han incluido el tema laboral dentro de sus regulaciones. En el plano nacional, la Constitución mexicana (1917) y la Constitución de Weimar (1919) son los primeros textos constitucionales que elevan los 
derechos laborales a la pirámide normativa nacional, propuesta que es recogida luego por la mayoría de las constituciones del siglo XX. Con ello se consolida la ubicación de los derechos laborales dentro del listado de los derechos humanos o de los derechos fundamentales.

La interrogante por dilucidar consiste en establecer cuáles son los derechos laborales recogidos en los instrumentos internacionales de derechos humanos. Para dar respuesta a esta interrogante partamos por reconocer que la dogmática jurídica internacional de los derechos humanos se divide entre los ámbitos universal y regional. Así, los instrumentos internacionales del sistema universal son la Declaración Universal de Derechos Humanos y los Pactos Internacionales de 1966. El ámbito regional se divide en tres áreas geográficas que han regulado los derechos humanos: interamericano, europeo y africano. En el ámbito regional americano tenemos la Carta de la Organización de Estados Americanos (1948), la Declaración Americana de los Derechos y Deberes del Hombre (1948), la Convención Americana de Derechos Humanos (1969) y el Protocolo Adicional de San Salvador (1988) ${ }^{6}$. En el ámbito regional europeo tenemos el Estatuto del Consejo de Europa (1949), el Convenio Europeo de los Derechos Humanos y Libertades Fundamentales (1950) y sus diversos Protocolos, la Carta Social Europea (1961) y sus Protocolos, además de la Carta Social Europea revisada (1996). En el ámbito regional africano tenemos la Carta Africana de Derechos Humanos y de los Pueblos (1981).

De esta manera, estos instrumentos internacionales nos permiten elaborar el listado de derechos laborales consagrados como derechos humanos, sin dejar de mencionar que también los derechos laborales son recogidos en otros tratados internacionales de derechos humanos, como los tratados de protección de personas y grupos vulnerables, algunos de los cuales son: la Convención Internacional sobre la Eliminación de todas las Formas de Discriminación Racial, la Declaración de los Derechos del Niño, la Convención sobre los Derechos del Niño, la Convención sobre Eliminación de todas las Formas de Discriminación contra la Mujer, la Convención Interamericana para Prevenir, Sancionar y Erradicar la Violencia contra la Mujer, la Convención Americana para la Eliminación de todas las Formas de Discriminación contra las Personas con Discapacidad, la Convención Internacional sobre la Protección de los Derechos de Todos los Trabajadores Migratorios y de sus Familiares.

El listado de los derechos humanos laborales es el siguiente:

- La libertad de trabajo se encuentra recogida en el artículo 23,1 de la Declaración Universal de Derechos Humanos; el artículo 6.1 del Pacto Internacional de Derechos Económicos, Sociales y

6 También se debe incluir en esta lista a la Carta Internacional Americana de las Garantías Sociales (1947), a pesar que se elaboró con anterioridad a la constitución del sistema interamericano de derechos humanos. 
Culturales; el artículo XIV de la Declaración Americana de los Derechos y Deberes del Hombre; el artículo 6.1 del Protocolo Adicional de San Salvador; el artículo 1.2 de la Carta Social Europea; el artículo 5,e,i) de la Convención Internacional para la Eliminación de todas las Formas de Discriminación Racial; el artículo 11.1.c) de la Convención Internacional sobre la Eliminación de todas las Formas de Discriminación contra la Mujer; los artículos 52 y 53 de la Convención Internacional sobre la Protección de los Derechos de todos los Trabajadores Migratorios y de sus Familiares.

- La prohibición de la esclavitud y de la servidumbre se encuentra recogida en el artículo 4 de la Declaración Universal de Derechos Humanos; los artículos 8.1 y 2 del Pacto Internacional de Derechos Civiles y Políticos; el artículo 4.1 del Convenio Europeo de los Derechos Humanos y Libertades Fundamentales; el artículo 6.1 de la Convención Americana sobre Derechos Humanos; el artículo 5 de la Carta Africana de Derechos Humanos y de los Pueblos; y en el artículo 11.1 de la Convención Internacional sobre la Protección de los Derechos de todos los Trabajadores Migratorios y de sus Familiares.

- La prohibición del trabajo forzoso u obligatorio se encuentra recogida en el artículo 8.3 del Pacto Internacional de Derechos Civiles y Políticos; los artículos 4.2 y 3 del Convenio Europeo de los Derechos Humanos y Libertades Fundamentales; el artículo 1.2 de la Carta Social Europea; los artículos 6.2 y 3 de la Convención Americana sobre Derechos Humanos; y en los artículos 11.2, 3 y 4 de la Convención Internacional sobre la Protección de los Derechos de todos los Trabajadores Migratorios y de sus Familiares.

- El derecho al trabajo se encuentra recogido en el artículo 23.1 de la Declaración Universal de Derechos Humanos; el artículo 6 del Pacto Internacional de Derechos Económicos, Sociales y Culturales; el artículo XIV de la Declaración Americana de los Derechos del Hombre; el artículo 6 del Protocolo Adicional de San Salvador; el artículo 1 de la Carta Social Europea; el artículo 15 de la Carta Africana de Derechos Humanos y de los Pueblos; el artículo 5,e,i) de la Convención Internacional para la Eliminación de todas las Formas de Discriminación Racial; y en el artículo 11.1.a) de la Convención Internacional sobre la Eliminación de todas las Formas de Discriminación contra la Mujer.

- La protección contra el desempleo se encuentra recogida en el artículo 23.1 de la Declaración Universal de Derechos Humanos; el artículo 6.2 del Pacto Internacional de Derechos Económicos, 
Sociales y Culturales; el artículo 6.2 del Protocolo Adicional de San Salvador; el artículo 1.1 de la Carta Social Europea; y en el artículo 5,e,i) de la Convención Internacional para la Eliminación de todas las Formas de Discriminación Racial.

- La protección contra el despido se encuentra recogida en el artículo 7.d) del Protocolo Adicional de San Salvador; el artículo 24 de la Carta Social Europea revisada; el artículo 11.1.c) y 2.a) de la Convención Internacional sobre la Eliminación de todas las Formas de Discriminación contra la Mujer; y en el artículo 54.1.a) de la Convención Internacional sobre la Protección de los Derechos de todos los Trabajadores Migratorios y de sus Familiares.

- La prohibición de todo tipo de discriminación se encuentra recogida en los artículos 2 y 7 de la Declaración Universal de Derechos Humanos; los artículos 2 y 26 del Pacto Internacional de Derechos Civiles y Políticos; los artículos 2.2 y 3 del Pacto Internacional de Derechos Económicos, Sociales y Culturales; el artículo II de la Declaración Americana de los Derechos del Hombre; los artículos 1 y 24 de la Convención Americana sobre Derechos Humanos; el artículo 3 del Protocolo Adicional de San Salvador; los artículos 1 y 14 del Convenio Europeo de los Derechos Humanos y Libertades Fundamentales; el artículo 1.2 de la Carta Social Europea y el artículo 1 del Protocolo Adicional de la Carta Social Europea de 1988; los artículos 20 y E de la Carta Social Europea revisada; los artículos 2 y 3 de la Carta Africana de Derechos Humanos y de los Pueblos; el artículo 5 de la Convención Internacional para la Eliminación de todas las Formas de Discriminación Racial; el artículo 11.1.b) de la Convención Internacional sobre la Eliminación de todas las Formas de Discriminación contra la Mujer; y en el artículo 25 de la Convención Internacional sobre la Protección de los Derechos de todos los Trabajadores Migratorios y de sus Familiares.

- El derecho a la igualdad de remuneración se encuentra recogido en el artículo 23.2 de la Declaración Universal de Derechos Humanos; el artículo 7,a,i) del Pacto Internacional de Derechos Económicos, Sociales y Culturales; el artículo 7.a) del Protocolo Adicional de San Salvador; el artículo 4.3 de la Carta Social Europea; el artículo 15 de la Carta Africana de Derechos Humanos y de los Pueblos; el artículo 5,e,i) de la Convención Internacional para la Eliminación de todas las Formas de Discriminación Racial; y en el artículo 11.1.d) de la Convención Internacional sobre la Eliminación de todas las Formas de Discriminación contra la Mujer. 
- Laprohibición a la discriminación de las personas con responsabilidades familiares se encuentra recogida en el artículo 27 de la Carta Social Europea revisada.

- El derecho a la seguridad e higiene en el trabajo se encuentra recogido en el artículo 7.b) del Pacto Internacional de Derechos Económicos, Sociales y Culturales; el artículo 7.e) del Protocolo Adicional de San Salvador; el artículo 3 de la Carta Social Europea; el artículo 11.1.f) de la Convención Internacional sobre la Eliminación de todas las Formas de Discriminación contra la Mujer; y en el artículo 25.1.a) de la Convención Internacional sobre la Protección de los Derechos de todos los Trabajadores Migratorios y de sus Familiares.

- El derecho a condiciones justas, equitativas y satisfactorias de trabajo se encuentra recogido en el artículo 23.1 de la Declaración Universal de Derechos Humanos; el artículo 7 del Pacto Internacional de Derechos Económicos, Sociales y Culturales; el artículo 7 del Protocolo Adicional de San Salvador; el artículo 2 de la Carta Social Europea; el artículo 15 de la Carta Africana de Derechos Humanos y de los Pueblos; el artículo 5.e.i) de la Convención Internacional para la Eliminación de todas las Formas de Discriminación Racial; el artículo 32.2.b) de la Convención de los Derechos del Niño; y en el artículo 25 de la Convención Internacional sobre la Protección de los Derechos de todos los Trabajadores Migratorios y de sus Familiares.

- El derecho a la limitación de la jornada máxima de trabajo se encuentra recogido en el artículo 24 de la Declaración Universal de Derechos Humanos; el artículo 7.d) del Pacto Internacional de Derechos Económicos, Sociales y Culturales; el artículo 7.g) del Protocolo Adicional de San Salvador; el artículo 2.1 de la Carta Social Europea; y en el artículo 25.1.a) de la Convención Internacional sobre la Protección de los Derechos de todos los Trabajadores Migratorios y de sus Familiares.

- El derecho al descanso semanal remunerado se encuentra recogido en el artículo 24 de la Declaración Universal de Derechos Humanos; el artículo 7.d) del Pacto Internacional de Derechos Económicos, Sociales y Culturales; el artículo XV de la Declaración Americana de los Derechos del Hombre; el artículo 7.h) del Protocolo Adicional de San Salvador; el artículo 2.5 de la Carta Social Europea; y en el artículo 25.1.a) de la Convención Internacional sobre la Protección de los Derechos de todos los Trabajadores Migratorios y de sus Familiares.

- El derecho al descanso remunerado en feriados se encuentra recogido en el artículo 7.d) del Pacto Internacional de Derechos

EL REDIMENSIONAMIENTO DEL DERECHO

INTERNACIONAL DEL TRABAJO

THE RESIZING OF INTERNATIONAL LABOR LAW 
Económicos, Sociales y Culturales; el artículo 7.h) del Protocolo Adicional de San Salvador; y en el artículo 2.2 de la Carta Social Europea.

- El derecho a vacaciones pagadas se encuentra recogido en el artículo 24 de la Declaración Universal de Derechos Humanos; el artículo 7.d) del Pacto Internacional de Derechos Económicos, Sociales y Culturales; el artículo 7.h) del Protocolo Adicional de San Salvador; el artículo 2.3 de la Carta Social Europea; el artículo 11.1.e) de la Convención Internacional sobre la Eliminación de todas las Formas de Discriminación contra la Mujer; y en el artículo 25.1.a) de la Convención Internacional sobre la Protección de los Derechos de todos los Trabajadores Migratorios y de sus Familiares.

- El derecho a una remuneración mínima se encuentra recogido en el artículo 23.3 de la Declaración Universal de Derechos Humanos; el artículo 7.a.ii) del Pacto Internacional de Derechos Económicos, Sociales y Culturales; el artículo 7.a) del Protocolo Adicional de San Salvador; el artículo 4.1 de la Carta Social Europea; y en el artículo 5.e.i) de la Convención Internacional para la Eliminación de todas las Formas de Discriminación Racial.

- El derecho a la promoción en el empleo se encuentra recogido en el artículo 7.c) del Pacto Internacional de Derechos Económicos, Sociales y Culturales; el artículo 7.c) del Protocolo Adicional de San Salvador; el artículo 1.d) del Protocolo Adicional de la Carta Social Europea de 1988; y en el artículo 11.1.c) de la Convención Internacional sobre la Eliminación de todas las Formas de Discriminación contra la Mujer.

- El derecho a la formación profesional se encuentra recogido en el artículo 6.2 del Pacto Internacional de Derechos Económicos, Sociales y Culturales; el artículo 6.2 del Protocolo Adicional de San Salvador; los artículos 1.4, 10 y 15 de la Carta Social Europea; y en el artículo 1.b) del Protocolo Adicional de la Carta Social Europea de 1988.

- El derecho a la información y a la consulta en el seno de la empresa se encuentra recogido en el artículo 2 del Protocolo Adicional de la Carta Social Europea de 1988 y en el artículo 21 de la Carta Social Europea revisada.

- El derecho a la información y a la consulta en los procedimientos de despido colectivo se encuentra recogido en el artículo 29 de la Carta Social Europea revisada.

- El derecho a la tutela de los créditos en caso de insolvencia de sus empleadores se encuentra recogido en el artículo 25 de la Carta Social Europea revisada. 
- La libertad sindical se encuentra recogida en el artículo 20 de la Declaración Universal de Derechos Humanos; el artículo 22.2 del Pacto Internacional de Derechos Civiles y Políticos; el artículo 8 del Pacto Internacional de Derechos Económicos, Sociales y Culturales; el artículo XXII de la Declaración Americana de los Derechos del Hombre; el artículo 16.1 de la Convención Americana sobre los Derechos Humanos; el artículo 8.1.a) del Protocolo Adicional de San Salvador; el artículo 11.1 del Convenio Europeo de los Derechos Humanos y Libertades Fundamentales; el artículo 5 de la Carta Social Europea; el artículo 10 de la Carta Africana de Derechos Humanos y de los Pueblos; el artículo 5.e.i) de la Convención Internacional para la Eliminación de todas las Formas de Discriminación Racial; el artículo 15 de la Convención de los Derechos del Niño; y en los artículos 26 y 40 de la Convención Internacional sobre la Protección de los Derechos de todos los Trabajadores Migratorios y de sus Familiares.

- El derecho a la protección de los representantes de los trabajadores contra actos que puedan causarles un perjuicio y de contar con las facilidades adecuadas para desempeñar sus funciones se encuentra recogido en el artículo 28 de la Carta Social Europea revisada.

- El derecho a la negociación colectiva se encuentra recogido en el artículo 6 de la Carta Social Europea y ampliado en el artículo 22 de la Carta Social Europea revisada.

- El derecho a la huelga se encuentra recogido en el artículo 8.d) del Pacto Internacional de Derechos Económicos, Sociales y Culturales; el artículo 8.1.b) del Protocolo Adicional de San Salvador; y en el artículo 6.4 de la Carta Social Europea.

- El derecho a la seguridad social se encuentra recogido en los artículos 22 y 25.1 de la Declaración Universal de Derechos Humanos; el artículo 9 del Pacto Internacional de Derechos Económicos, Sociales y Culturales; el artículo XVI de la Declaración Americana de los Derechos del Hombre; los artículos 9 y 10.2.d) del Protocolo Adicional de San Salvador; el artículo 12 de la Carta Social Europea; el artículo 11.1.e) de la Convención Internacional sobre la Eliminación de todas las Formas de Discriminación contra la Mujer; el artículo 26 de la Convención de los Derechos del Niño; y en los artículos 27 y 54.1.b) de la Convención Internacional sobre la Protección de los Derechos de todos los Trabajadores Migratorios y de sus Familiares.

- Los derechos de protección al menor de edad se encuentran recogidos en el artículo 24 del Pacto Internacional de Derechos 
Civiles y Políticos; los artículos 7.f) y 10.3 del Pacto Internacional de Derechos Económicos, Sociales y Culturales; el artículo VII de la Declaración Americana de los Derechos del Hombre; el artículo 19 de la Convención Americana sobre los Derechos Humanos; el artículo 16 del Protocolo Adicional de San Salvador; el artículo 7 de la Carta Social Europea; y en los artículos 19, 32, 34, 35 y 36 de la Convención de los Derechos del Niño.

- Los derechos a la protección de la mujer trabajadora se encuentran recogidos en el artículo 10.2 del Pacto Internacional de Derechos Económicos, Sociales y Culturales; el artículo VII de la Declaración Americana de los Derechos del Hombre; el artículo 9.2 del Protocolo Adicional de San Salvador; el artículo 8 de la Carta Social Europea; y en el artículo 11 de la Convención Internacional sobre la Eliminación de todas las Formas de Discriminación contra la Mujer.

- Los derechos de protección a los trabajadores migrantes se encuentran recogidos en el artículo 19 de la Carta Social Europea y en los artículos 7 al 63 de la Convención Internacional sobre la Protección de los Derechos de todos los Trabajadores Migratorios y de sus Familiares.

- Los derechos de protección a los trabajadores minusválidos se encuentran recogidos en el artículo 18.a) del Protocolo Adicional de San Salvador; el artículo 15 de la Carta Social Europea.

Este largo listado de treinta derechos laborales recogidos en instrumentos internacionales de derechos humanos muestra un amplio marco regulador de ese espacio común entre el DIT y el DIDH. La elevación de los derechos laborales a la categoría de derechos humanos conduce a una serie de consecuencias jurídicas importantes. En primer lugar, los ubica en la cúspide de la normativa internacional en materia de derechos. En segundo lugar, la ratificación de los instrumentos internacionales de derechos humanos genera una serie de obligaciones internacionales para los Estados. En tercer lugar, estos derechos se encuentran protegidos por los procedimientos de control internacional de los derechos humanos.

\section{LA PROTECCIÓN INTERNACIONAL DE LOS DERECHOS HUMANOS LABORALES}

Sería excesivamente extenso para el presente estudio revisar cada uno de los procedimientos de control internacional de los derechos humanos. Resulta más pertinente mencionar algunos pronunciamientos que muestren el valor agregado adquirido por los derechos laborales recogidos en los instrumentos internacionales de derechos humanos. 
Para ello se presenta una breve selección de pronunciamientos internacionales sobre casos resaltantes agrupados temáticamente en cinco materias laborales: la libertad sindical, la prohibición de la discriminación, la prohibición de la esclavitud, el derecho al trabajo y la seguridad e higiene en el trabajo. Estos pronunciamientos pueden marcar una pauta de la dimensión protectora de los derechos humanos laborales.

a. Libertad sindical: el caso «Delia Saldías de López vs. Uruguay» (Comité de Derechos Humanos) subraya que donde un trabajador y un líder sindical fueran objeto de diversas formas de hostigamiento por las autoridades desde el inicio de su implicación en el sindicato, incluyendo el arresto y la detención sin juicio, y sujeto a tortura y tratamiento cruel, inhumano o degradante, el derecho a la libertad sindical fue violado. El caso «Baena y Otros vs. Panamá» (Corte Interamericana de Derechos Humanos) señala que la libertad sindical consiste básicamente en la facultad de constituir organizaciones sindicales y poner en marcha su estructura interna, actividades y programas de acción sin intervención de las autoridades públicas que limite o entorpezca el ejercicio del respectivo derecho. Por otra parte, este derecho supone que cada persona pueda determinar sin coacción alguna, si desea o no formar parte de la asociación. Se trata, pues, del derecho fundamental de agruparse para la realización común de un fin lícito sin presiones o intromisiones que puedan alterar o desnaturalizar su finalidad.

b. Prohibición de la discriminación: El caso «Gaygusuz vs. Austria» (Corte Europea de Derechos Humanos) establece que un trato es discriminatorio si no tiene una justificación objetiva y razonable, esto es, si no persigue un «objetivo legítimo» o si no hay una «relación razonable de proporcionalidad entre los medios empleados y el objetivo intentado para ser observado». El Informe 2002 (Comisión Interamericana de Derechos Humanos) concluye que el Estado no puede discriminar en la protección y garantía de los derechos laborales fundamentales entre los trabajadores, con independencia de su estatus migratorio.

c. Prohibición de la esclavitud: el caso «Kunarac» (Tribunal Penal Internacional para la Antigua Yugoslavia) subraya que las formas contemporáneas de esclavitud forman parte de enslavement como un crimen contra la humanidad bajo el derecho consuetudinario internacional. El caso Barcelona «Traction» (Corte Internacional de Justicia) identifica la prohibición de la esclavitud como una obligación internacional erga omnes. 
d. El derecho al trabajo: la Observación General N. 18 (Comité de Derechos Económicos, Sociales y Culturales) sostiene que el derecho al trabajo es un derecho individual que pertenece a cada persona, y a la vez un derecho colectivo. Engloba todo tipo de trabajos, ya sean autónomos o trabajos dependientes sujetos a un salario. El derecho al trabajo no debe entenderse como un derecho absoluto e incondicional a obtener un empleo. La «Reclamación N. ${ }^{\circ} 6$ Sindicato de Trabajadores de Turismo vs. Francia» (Comité Europeo de Derechos Sociales) resalta que es obligación del Estado proteger efectivamente el derecho de los trabajadores a ganarse la vida mediante un empleo libremente elegido. Esta obligación requiere inter alia la eliminación de todas las formas de discriminación en el empleo cualquiera que sea la naturaleza legal de la relación profesional.

e. Seguridad e higiene en el trabajo: el caso «Amianto —Canadá vs. Francia— (Órgano de Apelación del Sistema de Solución de Diferencias de la $\mathrm{OMC}$ ) señala que no cabe discutir que los Estados Miembros de la OMC tienen derecho a determinar el nivel de protección sanitaria que consideren apropiada para una situación dada. Por lo que parece legítimo que un Miembro trate de defender la difusión de un producto que entrañe altos riesgos entre su población, incluyendo los trabajadores. Este breve recorrido de pronunciamientos nos muestra la dimensión de la protección internacional de los derechos humanos laborales, dejando a un lado la errónea idea de que el DIT se reduce al ámbito protector de la OIT. En realidad, los derechos laborales son protegidos en los diversos sistemas normativos del Derecho Internacional donde se encuentran recogidos.

\section{IV.REFLEXIÓN FINAL}

Podemos concluir que los derechos humanos laborales son un ordenamiento internacional existente -no por crear- que se encuentran protegidos por un conjunto de procedimientos de control y con pronunciamientos internacionales que tienen niveles de obligatoriedad para los Estados donde se ampara su protección.

En mi opinión, asistimos a un cambio de paradigma normativo que se traslada desde el Derecho del Trabajo hacia el DIT, donde ahora son los derechos humanos laborales los nuevos pilares de protección de la libertad en el mundo del trabajo. 